\title{
A NOVEL METHOD TO TRANSFER POROUS PDMS MEMBRANES FOR HIGH THROUGHPUT ORGAN-ON-CHIP AND LAB-ON-CHIP ASSEMBLY
}

\author{
W.F. Quirós-Solano ${ }^{1,4}$, N. Gaio ${ }^{1}$, C. Silvestri ${ }^{1}$, Y.B. Arik ${ }^{2}$, O.M.J.A. Stassen ${ }^{3}$, A.D. van der Meer ${ }^{2}$, \\ C.V.C. Bouten ${ }^{3}$, A. van den Berg ${ }^{2}$, R. Dekker ${ }^{l}$ and P.M. Sarro ${ }^{1}$ \\ ${ }^{1}$ Delft University of Technology, Delft, THE NETHERLANDS \\ ${ }^{2}$ University of Twente, Enschede, THE NETHERLANDS \\ ${ }^{3}$ Eindhoven University of Technology, Eindhoven, THE NETHERLANDS \\ ${ }^{4}$ Instituto Tecnológico de Costa Rica, Cartago, COSTA RICA
}

\begin{abstract}
We present a novel method to easily and reliably transfer highly porous, large area, thin microfabricated Polydimethylsiloxane (PDMS) porous membranes on Lab-on-Chip (LOC) and Organ-on-Chip (OOC) devices. The use of silicon as carrier substrate and a water-soluble sacrificial layer allows a simple and reproducible transfer of the membranes to any PDMS-based OOC and LOC device. The use of IC and MEMS compatible techniques reduces significantly the fabrication time and the need of manual handling. Our method is suitable for automatic assembling systems, such as pick-and-place, crucial to significantly increase the throughput of OOC and LOC devices assembling. Membranes with $8 \mu \mathrm{m}$ pore size and as thin as $4 \mu \mathrm{m}$ are successfully transferred. The viability and biocompatibility of the transfer was assessed by culturing two different cell lines on an OOC with transferred porous PDMS membranes.
\end{abstract}

\section{INTRODUCTION}

During the past three decades microfluidic technologies have enabled the manipulation and control of very small fluid volumes $(100 \quad \mathrm{~nL}-10 \mu \mathrm{L})$ using microfabricated structures. This has consequently made possible the maintenance and growth of cells within integrated and robust microenvironments known as Labon-Chip (LOC) devices.

Advances in tissue engineering on one side and microfabrication on the other, have brought cell biology studies to a significantly higher level, reinforced by the capability to realize microstructures that better recapitulate the intricate in vivo conditions of the human body [1]. Lately, such advances have opened up a new field, the so-called Organ-on-Chip (OOC) devices [2]. These devices are becoming a strong candidate to replace conventional static cell cultures and animal testing in drug development [3].

These customized microenvironments generally consist of an assembly of 3D structures fabricated by soft lithography. They often comprise a top and a bottom thick PDMS layers, hosting microfluidic channels interfaced by a porous polymeric membrane (Fig. 1) [4].

The porous polymeric membrane can play different roles in such devices. They can function as support for tissue differentiation, tissue organization and further allowing the recapitulation of tissue-tissue interfaces with co-cultures.

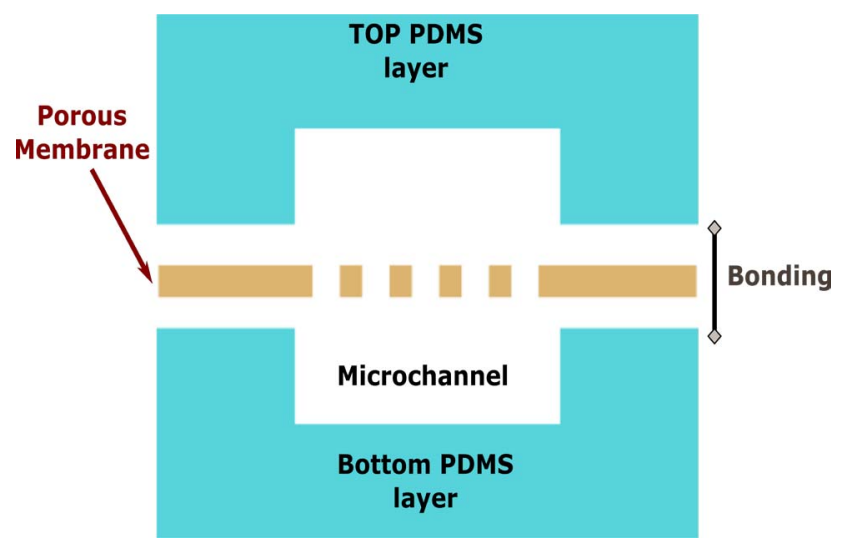

Figure 1: Typical LOC and OOC structure: A top and bottom PDMS layer hosting microchannels interfaced by a porous membrane.

Currently, these porous membranes for OOC devices are created either using PDMS, polyethylene terephthalate (PET) or polycarbonate (PC) $[5,6]$. However, the prevailing fabrication processes used to realize porous membranes with such materials intrinsically limit their applicability and impede the upscaling of the fabrication for the entire drug development cycle. In fact, porous PDMS membranes are often developed by replica molding, relying on manual fabrication procedures that are time-consuming and often causes peeling off (Fig. 2a). Moreover, features such as minimum pore size, thickness and porosity are limited and hard to tune with such a fabrication approach [7].

On the other hand, porous PET and PC membranes do not offer control on the distribution of the pores, the porosity range is limited and are cumbersome to attach to PDMS-based devices as they rely strongly on manual handling. Consequently, the required proper alignment and the resulting flat surface needed (Fig. 2b) are not easily assured.

Manual handling hampers high throughput and affects alignment and flatness, which might negatively influence the biological findings. Additionally, some of these materials are not as transparent and mechanically flexible when compared to PDMS. 


\section{Replica molding}

a)
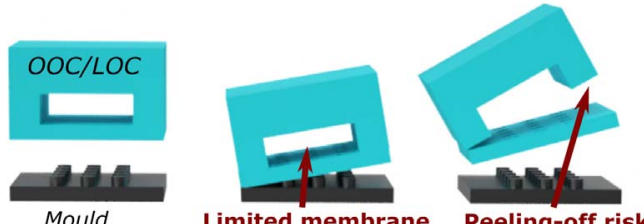

Limited membrane Peeling-off risk features

Manual attachment
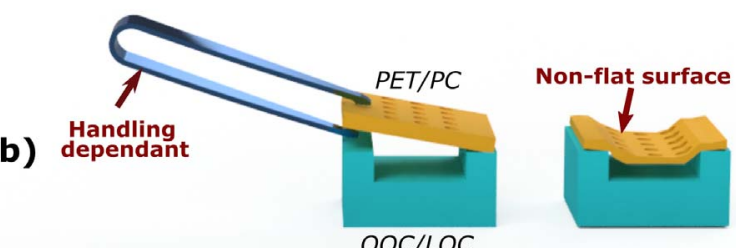

OOC $/$ LOC

Figure 2: The shortcomings of a) replica molding and b) manual attachment, the currently available methods for assembling of $L O C$ and $O O C$ devices with porous membranes.

Here, we propose a simple and reproducible method to transfer microfabricated porous PDMS membranes into LOC and OOC devices. It allows circumventing the limitation imposed by the manual handling of the conventional assembling methods to create porous membranes for such devices, while keeping the wellknown mechanical and optical advantages PDMS offers over other materials. Our method can be adapted to automated assembling techniques, such as pick-and-place, for high throughput, accurate alignment and reproducible assembling of LOC and OOC devices.

\section{MEMBRANES FABRICATION TRANSFER METHOD}

Fabrication of the porous PDMS

The fabrication process of the PDMS porous membranes is fully compatible with IC and MEMS technology. The porous membranes are fabricated at wafer level to subsequently transfer them to LOC and OOC devices (Fig. 2a).

The fabrication process uses Poly-acrylic acid (PAA) as sacrificial layer. PAA is known for its high solubility in water and has previously been demonstrated as sacrificial layer in 2D micromachining [8]. The process flow to pattern the porous PDMS membranes is illustrated in Figure 3. Briefly, a $1 \mu \mathrm{m}$ thick PAA layer is initially deposited on a $100 \mathrm{~mm}$ diameter silicon (Si) wafer (Fig. 3a-b) by spin coating. Then, a PDMS layer is deposited (Fig. 3c) and patterned through a customized lithography process and dry etching. The thickness of such PDMS layer can be tuned between $2 \mu \mathrm{m}$ and $50 \mu \mathrm{m}$ with $97.8 \%$ uniformity.

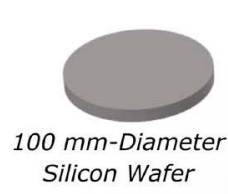

(a)

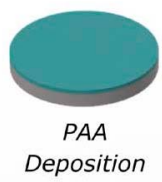

(b)

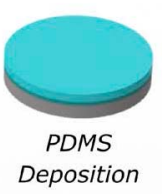

(c)

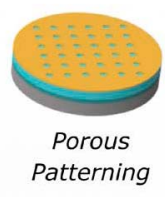

(d)
Figure 3: The main steps of the process flow developed to fabricate the porous PDMS membranes for LOC and OOC devices. (a-c) deposition of PAA, PDMS on Si substrate and (d) lithographic patterning of the pore arrays.

\section{Transferring of the porous PDMS}

Our method proposes a simple and reproducible way to transfer the microfabricated porous PDMS layer as membranes for LOC and OOC devices. The main steps of the transfer process are depicted in Figure 4. Firstly, both the thick PDMS bottom part of the PDMS device and the porous PDMS surface on the Si (carrier) wafer are treated with oxygen plasma to activate their surface and guarantee a successful mechanical bonding (Fig. 4a). Then, the carrier and the thick PDMS bottom part of the device are bonded together by applying a constant pressure $(73 \mathrm{kPa}$ ) for 2 hours (Fig. 4b). Thanks to the lithography based fabrication approach, localized porous

\section{$\begin{array}{llll}\text { Treatment } & \text { Bonding } & \text { Dicing } & \text { Releasing }\end{array}$}

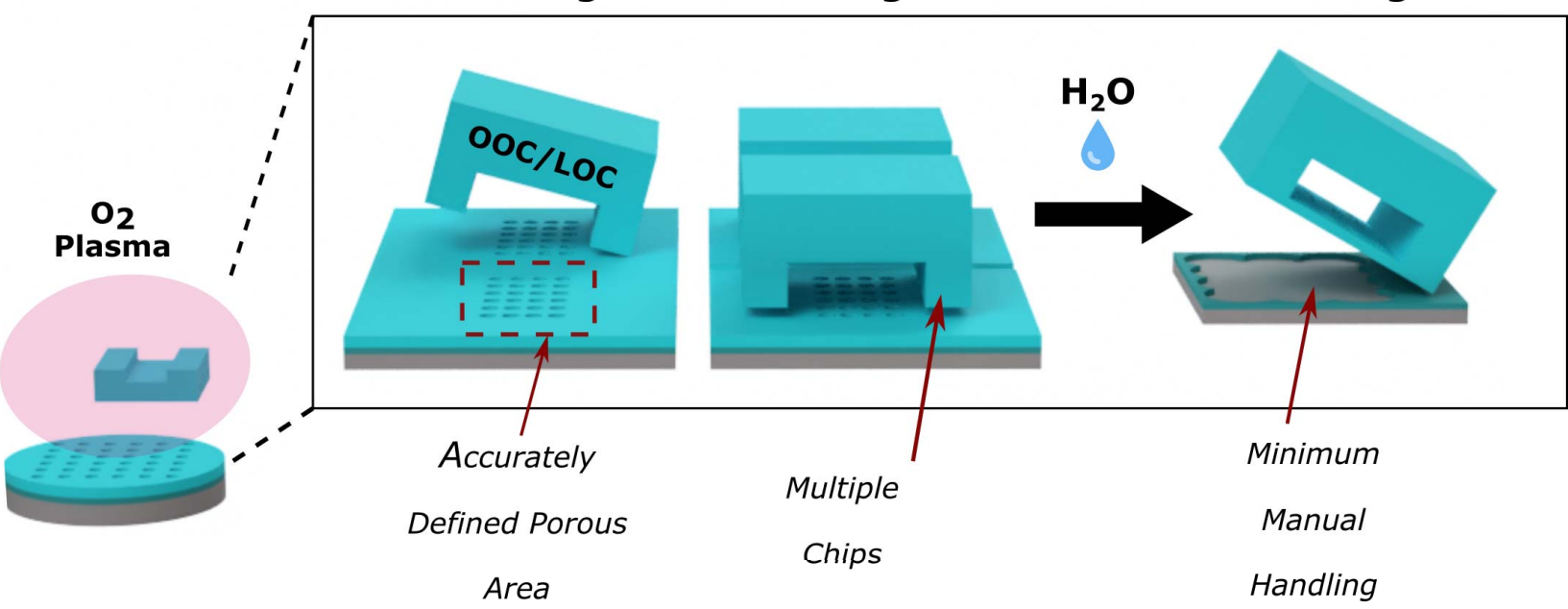

(a)

(b)

(c)

(d)

Figure 4: Main steps of the developed transfer method of porous PDMS membranes for LOC and OOC devices: (a) PDMS surface treatment, (b) Mechanical bonding, (c) Chip dicing and (d) Releasing. 
and non-porous regions can be specifically defined on the carrier substrate for any device size to enhance the mechanical bonding accordingly. The carrier containing the porous layer can therefore be diced to match the dimensions of different targeted LOC or OOC devices (Fig. 4c). To release the porous PDMS membrane from the carrier and keep them suspended on the bottom PDMS part, the assembled die is dipped in water using an ultrasonic bath. After approximately $15 \mathrm{~min}$, the PAA is dissolved and the PDMS detaches from the carrier (Fig. 4d) completing the transfer of the porous membrane. The final device is finalized by the placement of the top PDMS part. To do so, both the bottom part and the top part with the porous membrane are treated with oxygen plasma and bonded as previously described, completing the device assembly.

\section{RESULTS AND DISCUSSION}

The fabricated bottom part of one of the OOC device used in our experiments can be observed in Figure 5a. The device pictured is still attached to the silicon carrier, the stage prior to its release. This device was previously used by van der Helm [9] to directly measure transepithelial electrical resistance (TEER) to study cellular barrier tightness. In Figure 5b, a Scanning Electron Microscope (SEM) image the porous membranes transferred to this OOC device is shown. A $4 \mu \mathrm{m}$-thick membrane with well-defined pores of $8 \mu \mathrm{m}$-diameter was successfully transferred demonstrating the applicability of the method to thin PDMS porous membranes $(<10 \mu \mathrm{m})$. Membranes of different thicknesses can be also transferred according to the application requirements.

By relying on a bulk carrier substrate compatible with IC and MEMS fabrication techniques, our process guarantees a good reproducibility and flat transferred membranes. This method can be considered suitable for automated assembling techniques, such as pick-and-place, since for several steps we only need to bring surfaces in close contact, an operation that is achievable with robotic technologies available in automated assembling systems. Therefore, our method enables the envisioned highthroughput and reproducible assembling of PDMS-based OOC and LOC devices.

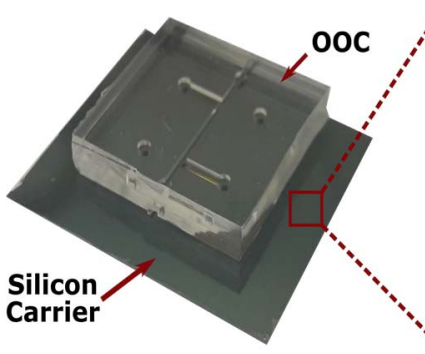

(a)

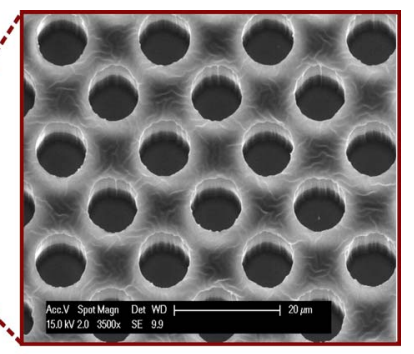

(b)
Figure 5: Optical and SEM images of the fabricated porous PDMS and the OOC device. (a) The silicon carrier containing the 4- $\mu \mathrm{m}$ thick porous PDMS bonded to the OOC device. (b) A SEM image of the fabricated porous membranes with $8 \mu \mathrm{m}$ diameter pore size.
To evaluate the validity and biocompatibility of the process and transfer method, both Human Umbilical Vein Endothelial cells (HUVECs) and Vascular Interstitial cells (VICs) were cultured on the transferred membranes. In Figure $6 \mathrm{a}$, a complete OOC device after transferring the membranes and prior to cell seeding is shown. In this specific case, a chip with a $25 \%$ porosity membrane is shown. As clearly shown in Figure 6b, flat and clean membranes were successfully transferred.

Once the transfer was completed, prior to cell seeding in one of the microchannels, the surface of the microchannel was functionalized with APTES and glutaraldehyde and rinsed with PBS and coated with 0.1 $\mathrm{mg} / \mathrm{ml}$ collagen. Subsequently, cells were attached by incubating the chips for $30 \mathrm{~min}$. In Figure $6 \mathrm{c}$, a fluorescence microscopy image of the cells cultured on the porous membranes, is reported. The morphology of the cells, for such incubating time, suggested a healthy environment for cell growth with no risks of infection imposed by the porous membranes or any possible residue from the transfer process.

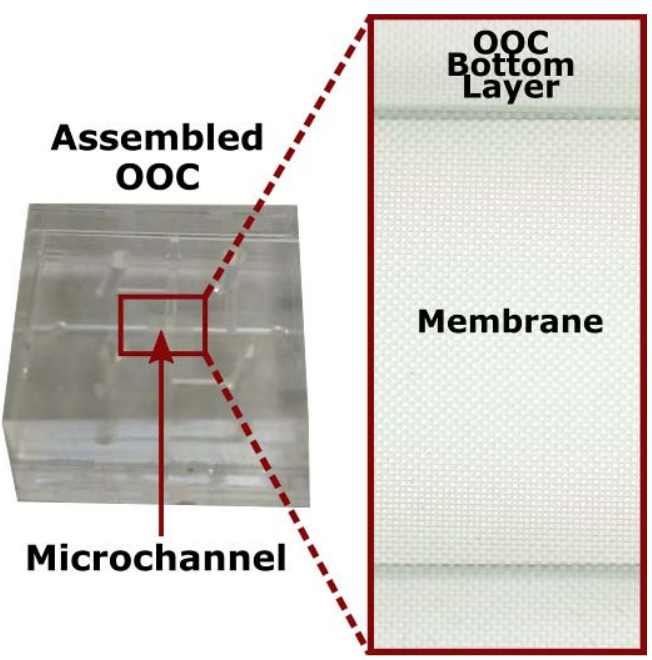

(a)

(b)

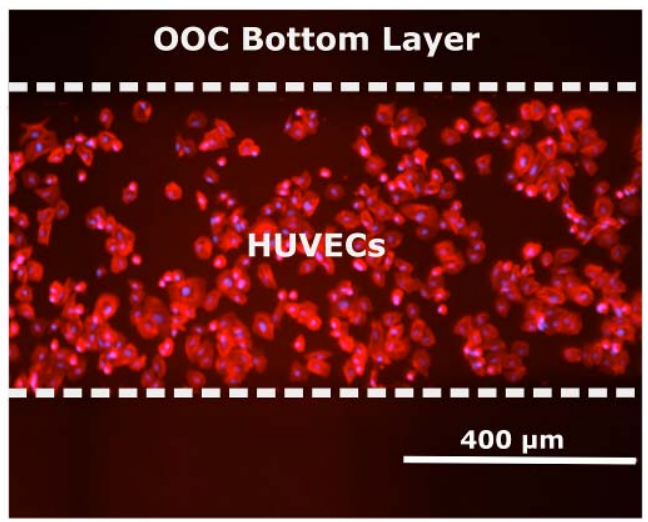

(c)

Figure 6: Optical images of: (a) the fully assembled OOC device; (b) a close-up of the microchannel area with the transferred porous membrane and (c) a fluorescent image of HUVECS cultures on the PDMS membranes. 
Similarly, to demonstrate the viability of culturing more than one cell line, porous membranes were transferred and both HUVECs and VICs were co-cultured in a same device (Fig. 7b). VICs were cultured on the bottom microchannel, and similarly as the previous experiment; HUVECs were cultured on the top channel. The porous membranes create an artificial interface between both cell types. In Figure $7 \mathrm{c}$, a florescence microscopy image obtained through confocal microscope show the cells on both sides of the porous membranes seeded in the corresponding microchannels. Both, the HUVECs and VICs in contact with the membranes showed healthy morphology, demonstrating once again the validity of the method to develop and transfer biocompatible PDMS porous membranes on OOC devices.

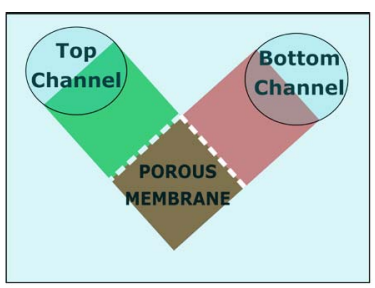

(a)

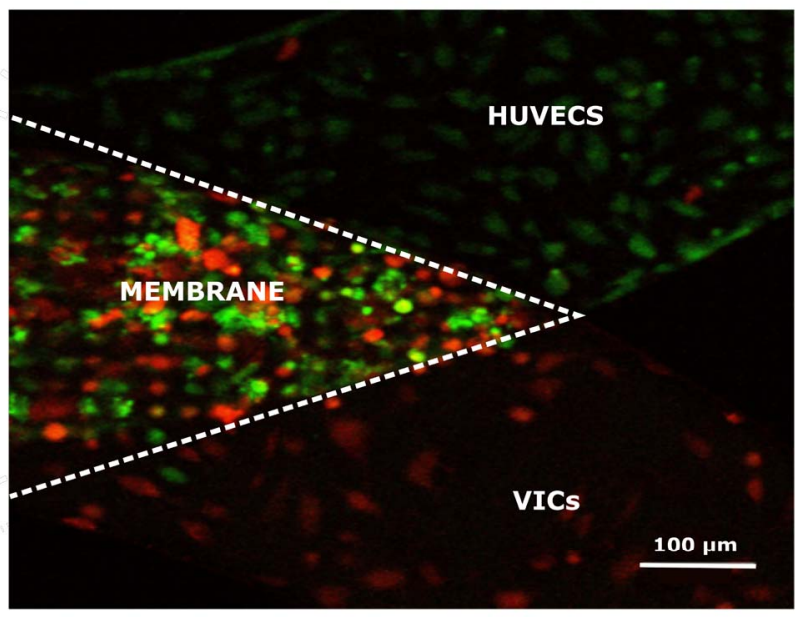

(c)

Figure 7: Conceptual drawing and microscope images of the OOC device, and HUVECs and VICs seeded on an OOC device. (a) Top view of the device, (b) Cross section of the device, (c) fluorescence confocal images of the cells cultured on a membrane transferred to a OOC device.

\section{CONCLUSION}

We demonstrated a novel method to easily and reliably transfer porous PDMS membranes into OOC devices. The porous PDMS membranes are fully fabricated at silicon wafer scale, which in combination with the water-soluble polymer PAA allows to significantly simplify the assembly of LOC and OOC devices, while providing a high yield and high reproducibility. The compatibility with IC and MEMS techniques allows to adapt the process to automated assembling techniques, to bring the assembly of OOC and LOC devices a step further towards reproducible and high throughput manufacturing.
Finally, it was proven that our novel method does not disturb the viability of cell culturing. This was demonstrated by two different cell lines cultured on OOC devices with transferred thin porous PDMS membranes which showed healthy morphology.

\section{ACKNOWLEDGEMENTS}

The authors gratefully acknowledge the technical support and advice of the staff at the Else Kooi Lab, MIRA and BME laboratories at TU/e. This work was a pilot project under the framework of the NanoInside: Organs-on-Chips Technology Platform, a subsidy promoted by hDMT and granted by NanoNextNL.

William F. Quirós-Solano is partially financed by Instituto Tecnológico de Costa Rica. Nikolas Gaio is financed by Electronic Components and Systems for European Leadership (ECSEL) "InForMed" (No.2014-2662155).

\section{REFERENCES}

[1] F. Lautenschläger and M. Piel, "Microfabricated devices for cell biology: all for one and one for all.," Curr. Opin. Cell Biol., vol. 25, no. 1, pp. 116-24, Feb. 2013.

[2] A. van de Stolpe and J. den Toonder, "Workshop meeting report Organs-on-Chips: human disease models.," Lab Chip, vol. 13, no. 18, pp. 3449-70, Sep. 2013.

[3] A. Polini, L. Prodanov, N. S. Bhise, V. Manoharan, M. R. Dokmeci, and A. Khademhosseini, "Organson-a-chip: a new tool for drug discovery.," Expert Opin. Drug Discov., vol. 9, pp. 335-52, 2014.

[4] D. Huh, Y. Torisawa, G. A. Hamilton, H. J. Kim, and D. E. Ingber, "Microengineered physiological biomimicry: Organs-on-Chips," Lab on a Chip, vol. 12. p. 2156, 2012.

[5] R. Booth and H. Kim, "Characterization of a microfluidic in vitro model of the blood-brain barrier ( $\mu$ BBB)," Lab Chip, vol. 12, no. 10, p. 1784, 2012.

[6] A. K. H. Achyuta, A. J. Conway, R. B. Crouse, E. C. Bannister, R. N. Lee, C. P. Katnik, A. A. Behensky, J. Cuevas, and S. S. Sundaram, "A modular approach to create a neurovascular unit-on-a-chip," Lab Chip, vol. 13, no. 4, pp. 542-553, 2013.

[7] Y. Xia and G. M. Whitesides, "Soft lithography," Annu. Rev. Mater. Sci., vol. 28, no. 12, pp. 153-184, 1998.

[8] V. Linder, B. D. Gates, D. Ryan, B. A. Parviz, and G. M. Whitesides, "Water-soluble sacrificial layers for surface micromachining," Small, vol. 1, no. 7, pp. 730-736, 2005.

[9] M. W. van der Helm, M. Odijk, J. P. Frimat, A. D. van der Meer, J. C. T. Eijkel, A. van den Berg, and L. I. Segerink, "Direct quantification of transendothelial electrical resistance in organs-on-chips," Biosens. Bioelectron., vol. 85, pp. 924-929, 2016.

\section{CONTACT}

*William F. Quirós-Solano, tel: +31625263062; w.f.quirossolano@tudelft.nl 